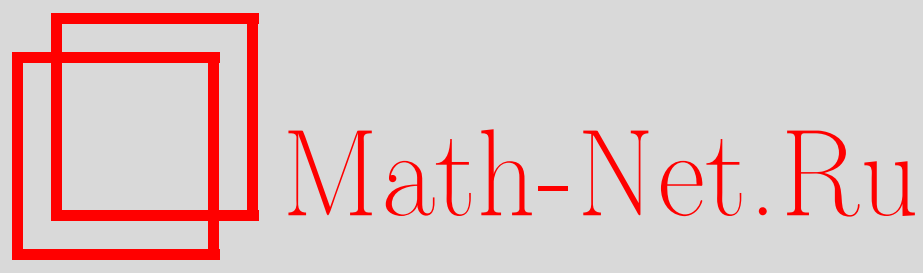

А. О. Барвинский, Туннелирующее космологическое состояние и происхождение хиггсовской инфляции в Стандартной модели, ТМФ, 2012, том 170, номер 1, 62-86

DOI: https://doi.org/10.4213/tmf6746

Использование Общероссийского математического портала Math-Net.Ru подразумевает, что вы прочитали и согласны с пользовательским соглашением http://www . mathnet.ru/rus/agreement

Параметры загрузки :

IP : 54.197 .130 .99

26 апреля 2023 г., 13:46:23

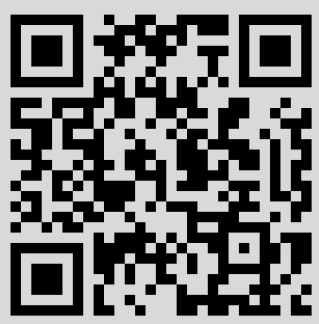




\title{
ТУННЕЛИРУЮЩЕЕ КОСМОЛОГИЧЕСКОЕ СОСТОЯНИЕ И ПРОИСХОЖДЕНИЕ ХИГГСОВСКОЙ ИНФЛЯЦИИ В СТАНДАРТНОЙ МОДЕЛИ
}

\begin{abstract}
Предложена формулировка интеграла по путям для туннелирующего космологического состояния, которая допускает непротиворечивую процедуру перенормировки и ренормгрупповое суммирование в физике частиц в квантовой космологии с тяжелыми массивными квантовыми полями. Эта формулировка применяется в инфляционной космологии, генерируемой бозоном Хиггса Стандартной модели, играющим роль инфлатона с сильной неминимальной связью с гравитацией. Получен полный космологический сценарий, который охватывает формирование начальных данных для инфляционного гравитационного фона в форме острого вероятностного пика в распределении поля инфлатона и последующую генерацию спектра космического микроволнового излучения на этом фоне. Также обсуждается статус космологического состояния Хартла-Хокинга и туннелирующего состояния в космологии безмассовых полей, конформно взаимодействующих с гравитацией.
\end{abstract}

Ключевые слова: квантовая гравитация и космология, инфляция, Стандартная модель, бозон Хиггса.

\section{1. ВВЕДЕНИЕ}

На заре инфляционной теории как источник начальных условий инфляции серьезно рассматривались два предписания для квантового состояния вселенной так называемые космологическая волновая функция Хартла-Хокинга [1] и волновая функция туннелирующего состояния [2], [3] (см. также обзор [4]), квазиклассические амплитуды которых обратно пропорциональны друг другу. В модели хаотической инфляции, генерируемой в приближении медленного скатывания полем инфлатона $\varphi$ с потенциалом $V(\varphi)$, эти амплитуды выглядят как $\left|\Psi_{ \pm}(\varphi)\right| \simeq e^{\mp S_{\mathrm{E}}(\varphi) / 2}$, где знак плюс отвечает волновой функции Хартла-Хокинга, а знак минус - туннелирующей волновой функции. Качественно обе эти функции описывают отщепление (квази)деситтеровского пространства-времени от евклидова полуинстантона, как это изображено на рис. 1 . Здесь $S_{\mathrm{E}}(\varphi)$ является евклидовым эйнштейновским

*Физический институт им. П. Н. Лебедева РАН, Москва, Россия. E-mail: barvin@td.lpi.ru 


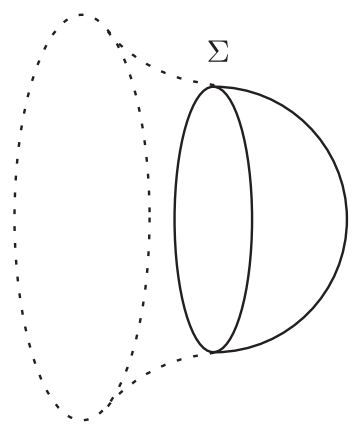

Рис. 1. Отщепление (квази)деситтеровского пространства-времени с лоренцевой сигнатурой, обозначенного штриховыми линиями, от полусферы евклидового гравитационного инстантона на трехмерной поверхности $\Sigma$.

действием полного деситтеровского инстантона $S^{4}$ с эффективной космологической постоянной, задаваемой значением поля инфлатона $\Lambda_{\mathrm{eff}}=V(\varphi) / M_{\mathrm{P}}^{2}$,

$$
S_{\mathrm{E}}(\varphi) \simeq-\frac{24 \pi^{2} M_{\mathrm{P}}^{4}}{V(\varphi)},
$$

в единицах редуцированной массы Планка $M_{\mathrm{P}}^{2}=1 / 8 \pi G$ (мы полагаем $\hbar=1=c$ ). Состояние Хартла-Хокинга было впервые сформулировано в виде функционального интеграла по евклидовым 4-геометриям; туннелирующее состояние в форме интеграла по лоренцевым метрикам было предложено в работах [3], [5]. Обе волновые функции были также получены как решения уравнения Уилера-де Витта в минисупепространстве.

Состояние Хартла-Хокинга и туннелирующее состояние приводят к противоположным физическим выводам. В частности, в силу отрицательного значения евклидового действия на деситтеровском инстантоне состояние Хартла-Хокинга увеличивает вклад вакуумных вселенных с $V(\varphi) \rightarrow+0$ и, таким образом, приводит к противоречащему интуитивным представлениям выводу, что бесконечно большие вселенные бесконечно более вероятны, чем вселенные конечного размера. Это свойство лежит в основе когда-то очень популярного, но сейчас почти забытого механизма Коулмена фиксации констант взаимодействия в квантовой гравитации [6]. С другой стороны, туннелирующее состояние благоприятствует большим значениям $V(\varphi)$, способным генерировать инфляционные сценарии. Таким образом, кажется, что туннелирующее состояние является более предпочтительным, чем состояние Хартла-Хокинга. Однако статус туннелирующего состояния оказывается не таким простым и даже является противоречивым.

Наивные попытки выйти за пределы приближения минисуперпространства приводят к ненормируемым состояниям в секторе пространственно-неоднородных степеней свободы материи и показывают несостоятельность обычного поворота Вика от лоренцевой сигнатуры пространства-времени к евклидовой. Эта проблема была частично преодолена путем наложения условия нормируемости на решение уравнения Уилера-де Витта [7], но ситуация осталась противоречивой по следующей 
причине. Отвлекаясь от проблемы интерференции между “сжимающейся" и "расширяющейся" ветвями космологической волновой функции, обсуждавшейся, например, в работах [4], [7]-[9], можно сказать, что амплитуды ветви Хартла-Хокинга и туннелирующей ветви такого квазиклассического решения имеют вид

$$
\left|\Psi_{ \pm}(\varphi, \Phi(\mathbf{x}))\right|=e^{\mp S_{\mathrm{E}}(\varphi) / 2}\left|\Psi_{\text {matter }}(\varphi, \Phi(\mathbf{x}))\right|
$$

где $\Phi(\mathbf{x})$ представляет собой набор материальных полей, отличных от пространственно однородного инфлатона, а $\Psi_{\text {matter }}(\varphi, \Phi(\mathbf{x}))$ - их нормируемая (квазигауссова) часть полной волновой функции, фактически представляющая евклидов деситтеровски-инвариантный вакуум линеаризованных полей $\Phi(\mathbf{x})$ на квазидеситтеровском фоне с космологической константой $\Lambda_{\mathrm{eff}}=V(\varphi) / M_{\mathrm{P}}^{2}$. Квантовое усреднение по $\Phi(\mathbf{x})$ приводит к следующему распределению поля инфлатона:

$$
\rho_{ \pm}^{1-\operatorname{loop}}(\varphi)=\int d[\Phi(\mathbf{x})]\left|\Psi_{ \pm}(\varphi, \Phi(\mathbf{x}))\right|^{2}=e^{\mp S_{\mathrm{E}}(\varphi)-S_{\mathrm{E}}^{1-\mathrm{loop}}(\varphi)}
$$

где

$$
S_{\mathrm{E}}^{1-\operatorname{loop}}(\varphi)=\frac{1}{2} \operatorname{Tr} \ln \frac{\delta^{2} S_{\mathrm{E}}[\varphi, \Phi]}{\delta \Phi(x) \delta \Phi(y)}
$$

есть вклад ультрафиолетово-расходящегося однопетлевого эффективного действия $[10]-[12]^{1)}$. С помощью этого алгоритма был получен острый вероятностный пик в туннелирующем распределении $\rho_{-}^{1-\operatorname{loop}}(\varphi)$ для модели с сильной неминимальной связью инфлатона с гравитацией [10], [13], [14]. Этот пик был интерпретирован как генерирующий квантовый масштаб инфляции, который представляет собой начальное условие инфляционного сценария. Замечательно, что по случайным причинам данный результат был свободен от обычного ультрафиолетового перенормировочного произвола. Он не требовал применения перенормировочной схемы поглощения ультрафиолетовых расходимостей путем переопределения констант взаимодействия в древесном действии $S_{\mathrm{E}}(\varphi)$.

Однако выше однопетлевого приближения и для других физических корреляторов ситуация меняется, и ультрафиолетовая перенормировка требуется в полном объеме, но в силу разных знаков $\mp S_{\mathrm{E}}(\varphi)$ в (2) эта перенормировка различна для туннелирующего состояния и состояния Хартла-Хокинга. Например, в случае состояния Хартла-Хокинга (ассоциируемого с обычным поворотом Вика к евклидову пространству-времени) теория является асимптотически-свободной, но она не будет асимптотически-свободной в туннелирующем случае. Туннелирующая версия теории противоречит базовым теоретико-полевым результатам в плоском пространстве-времени. Это делает очень противоречивой наивную конструкцию туннелирующего состояния вышеуказанного типа, в частности не позволяет выйти за пределы однопетлевого приближения и осуществить его ренормгрупповое (РГ) улучшение.

\footnotetext{
1) Для туннелирующего состояния уравнение (3) может рассматриваться как результат тонкого подбора: чтобы гарантировать справедливость этого уравнения, волновые функции оператора $\delta^{2} S_{\mathrm{E}} / \delta \Phi \delta \Phi$, в отличие от случая состояния Хартла-Хокинга, не должны быть регулярными на полюсе полусферы на рис. 1. Это условие регулярности дает естественный критерий отбора евклидового деситтеровски-инвариантного вакуума в рамках конструкции Хартла-Хокинга. Я благодарен В. А. Рубакову за обсуждение этого вопроса.
} 
В настоящей работе мы предлагаем решение проблемы путем формулировки нового предписания интеграла по путям для туннелирующего состояния вселенной. Эта формулировка основана на недавно предложенной конструкции космологической матрицы плотности [15], которая описывает микроканонический ансамбль космологических моделей [16]. Статистическая сумма этого ансамбля была вычислена в пространственно-замкнутой модели с произвольным набором скалярных, спинорных и векторных полей, конформно связанных с гравитацией. Она была получена в приближении седловой точки с доминирующим вкладом термических космологических инстантонов с топологией $S^{3} \times S^{1}$. Эти инстантоны включают также вакуумную топологию $S^{4}$, рассматриваемую как предельный случай, когда компактифицированное временно́е измерение $S^{1}$ в $S^{3} \times S^{1}$ оказывается разорванным в процессе перехода от $S^{3} \times S^{1}$ к $S^{4}$. Этот предельный переход в точности восстанавливает состояние Хартла-Хокинга [1], так что вся конструкция из работ [15], [16] может рассматриваться как обобщение вакуумного состояния Хартла-Хокинга на квазитепловой ансамбль. Основные физические выводы для этого ансамбля заключаются в том, что он существует в ограниченном интервале значений эффективной космологической постоянной, способен генерировать сценарий большого буста для космологического ускорения [17], и его вакуумный хартл-хокинговский элемент фактически не вносит вклада в статистическую сумму, потому что он подавлен бесконечным положительным значением своего действия. Это оригинальный эффект конформной аномалии квантовых полей [18], [19], которая качественно меняет древесное действие теории (1).

Далее мы показываем, что интеграл по путям указанного типа имеет другую седловую точку, соответствующую отрицательному значению функции хода $N<0$, которое калибровочно неэквивалентно значению $N>0$ [20]. В случае тяжелых массивных квантовых полей, генерирующих инфляцию, это приводит к инверсии знака действия в показателе экспоненты статистической суммы, следовательно, эта ситуация заслуживает название "туннелирующей”. В таком туннелирующем состоянии тепловой вклад исчезает, и его инстантон оказывается чисто вакуумным. И наконец, эта конструкция более не страдает от упомянутого выше противоречия с перенормировкой. Предполагается, что полное квантовое эффективное действие в рамках градиентного разложения и разложения по степеням кривизны вычисляется и перенормируется обычным набором контрчленов на фоне метрики общего типа. Затем результат следует аналитически продолжить в область $N<0$ и вычислить в туннелирующей седловой точке функционального интеграла по функции хода $N$. С другой стороны, мы показываем, что для космологии, генерируемой конформной теорией поля (CFT), в отличие от той, которая генерируется массивными полями, туннелирующее состояние запрещено на динамическом уровне.

Ниже мы применяем данную конструкцию к космологической модели, в которой лагранжиан гравитон-инфлатонного сектора имеет вид

$$
\begin{gathered}
\mathbf{L}\left(g_{\mu \nu}, \Phi\right)=\frac{1}{2}\left(M_{\mathrm{P}}^{2}+\xi|\Phi|^{2}\right) R-\frac{1}{2}|\nabla \Phi|^{2}-V(|\Phi|), \\
V(|\Phi|)=\frac{\lambda}{4}\left(|\Phi|^{2}-v^{2}\right)^{2}, \quad|\Phi|^{2}=\Phi^{\dagger} \Phi,
\end{gathered}
$$

где $\Phi$ - бозон Хиггса Стандартной модели (CM), среднее значение которого играет роль инфлатона и который обладает сильной неминимальной связью с кривизной

3 Теоретическая и математическая физика, т. 170, № 1, 2012 г. 
при $\xi \gg 1$. Здесь, как и выше, $M_{\mathrm{P}}$ - редуцированная масса Планка, $\lambda$ - константа четвертичного самодействия бозона $\Phi$, а $v$ представляет собой масштаб электрослабого (EW) нарушения симметрии.

Изначально мотивацией для изучения этой модели с бозоном $\Phi$ GUT-типа (GUT теория великого объединения) [21], [22] была задача избежать чрезмерно малого значения константы $\lambda$ путем привлечения неминимального взаимодействия с большой константой $\xi$. Позднее эта модель была поддержана надеждой генерировать начальные условия Хартла-Хокинга или туннелирующие начальные данные для инфляции [13], [14]. Эта же модель, но с бозоном Хиггса СM Ф вместо абстрактной постановки GUT [13], [14] была рассмотрена в работе [23], расширена до однопетлевого уровня в работе [24] и изучена с точки зрения механизма постинфляционного разогрева в работе [25]. РГ-улучшение в этой модели предсказало параметры космического микроволнового излучения (CMB), амплитуду и спектральный индекс, совместные с данными космического зонда WMAP в конечном интервале значений массы бозона Хиггса, близком к принятой большинством исследователей области, которая определяется условиями стабильности EW-вакуума и ограничениями теории возмущений [26]-[31].

Целью настоящей работы является обобщение результатов работ [29], [30], в рамках которого возникают новые свойства данной модели: она не только имеет совместный с данными зонда WMAP спектр возмущений CMB, но также может генерировать начальные условия для инфляционного решения, на фоне которого эти возмущения распространяются. Данные начальные условия реализуются в форме острого вероятностного пика в туннелирующей функции распределения инфлатона.

\section{2. ТУННЕЛИРУЮЩАЯ КОСМОЛОГИЧЕСКАЯ ВОЛНОВАЯ ФУНКЦИЯ В ФОРМУЛИРОВКЕ ИНТЕГРАЛА ПО ПУТЯМ}

Микроканоническая матрица плотности в квантовой космологии была предложена в работе [16] как формальный проектор на подпространство физических состояний, удовлетворяющих системе уравнений Уилера-де Витта $\widehat{H}_{\mu}(\varphi, \partial / i \partial \varphi) \rho\left(\varphi, \varphi_{-}\right)=0$ :

$$
\hat{\rho} \sim\left(\prod_{\mu} \delta\left(\widehat{H}_{\mu}\right)\right)
$$

где $\widehat{H}_{\mu}=H_{\mu}(q, p)$ обозначает операторную реализацию полного набора гравитационных гамильтоновой и импульсных связей, конденсированный индекс которых содержит как набор дискретных значений, так и непрерывные пространственные координаты, $\mu=(\perp, a, \mathbf{x}), a=1,2,3$. Фазовые переменные $q, p$ включают в себя набор пространственных метрических коэффициентов и материальных полей $q=\left(g_{a b}(\mathbf{x}), \phi(\mathbf{x})\right)$ (также обозначаемых через $\varphi$, когда они используются как аргументы ядра матрицы плотности) и их сопряженные импульсы $p$.

Обоснование матрицы (5) как матрицы плотности микроканонического ансамбля в пространственно-замкнутой космологии было выдвинуто в работе [16] на основе аналогии с системой без связей, обладающей сохраняющимся гамильтонианом $\widehat{H}$. Микроканоническое состояние с фиксированной энергией $E$ для такой системы задается матрицей плотности $\hat{\rho} \sim \delta(\widehat{H}-E)$. Главное отличие матрицы плотности (5) 
от этого случая заключается в том, что замкнутая космология не имеет свободно фиксируемых констант движения, как энергия или другие глобальные заряды. В качестве интегралов движения она обладает гамильтоновой и импульсными связями $H_{\mu}$, имеющими частное нулевое значение, поэтому выражение (5) можно рассматривать как аналог равнораспределения - естественный кандидат на микроканоническое квантовое состояние закрытой вселенной.

Пертурбативно (по крайней мере в квазиклассическом петлевом разложении) ядро этого проектора может быть записано как интеграл по путям в фазовом пространстве канонически квантуемой теории гравитации:

$$
\rho\left(\varphi_{+}, \varphi_{-}\right)=e^{\Gamma} \int_{q\left(t_{ \pm}\right)=\varphi_{ \pm}} D[q, p, N] \exp \left[i \int_{t_{-}}^{t_{+}} d t\left(p \dot{q}-N^{\mu} H_{\mu}\right)\right] .
$$

Здесь $N^{\mu}$ представляют собой лагранжевы множители, дуальные к связям, - функции хода и сдвига $N^{\mu}=\left(N(\mathbf{x}), N^{a}(\mathbf{x})\right)$, а функциональное интегрирование идет по историям, интерполирующим между конфигурациями $\varphi_{ \pm}$, которые являются аргументами матрицы плотности. Область интегрирования по $N^{\mu}$, разумеется, является вещественной, потому что интегрирование по лагранжевым множителям устроено так, чтобы породить дельта-функции связей. Гамильтоново действие в показателе экспоненты есть интеграл по координатному времени $t$, это действие является всего лишь параметром упорядочения, изменяющимся между произвольными начальным значением $t_{-}$и конечным значением $t_{+}$, причем результат совершенно не зависит от их выбора. Мера интегрирования $D[q, p, N]$, конечно, включает процедуру фиксации калибровки Фаддеева-Попова, которая делает весь интеграл не зависящим от выбора калибровки и параметризации времени.

После интегрирования по каноническим импульсам интеграл по путям принимает лагранжеву форму интеграла по координатам конфигурационного пространства $q$ и функциям хода и сдвига $N^{\mu}$. В совокупности они образуют пространственно-временную метрику

$$
d s^{2}=-N_{\text {Lorentz }}^{2} d t^{2}+g_{a b}\left(d x^{a}+N^{a} d t\right)\left(d x^{b}+N^{b} d t\right)
$$

с лоренцевой сигнатурой $g_{\mu \nu}^{\text {Lorentz }}$ и материальные поля $\phi$, в терминах которых лагранжева форма классического действия есть $S\left[g_{\mu \nu}^{\text {Lorentz }}, \phi\right]$. Сделать еще один шаг в выборе обозначений позволяет наблюдение, что эта лоренцева метрика может рассматриваться как евклидова метрика $g_{\mu \nu}^{\text {Euclid }}$ с мнимым значением евклидовой функции хода,

$$
d s^{2}=N_{\text {Euclid }}^{2} d t^{2}+g_{a b}\left(d x^{a}+N^{a} d t\right)\left(d x^{b}+N^{b} d t\right), \quad N_{\text {Lorentz }}=-i N_{\text {Euclid }},
$$

так что действие евклидовой теории связано с лоренцевым действием $S\left[g_{\mu \nu}^{\text {Lorentz }}, \phi\right]$ посредством типичного соотношения

$$
i S\left[g_{\mu \nu}^{\text {Lorentz }}, \phi\right]=-S_{\mathrm{E}}\left[g_{\mu \nu}^{\text {Euclid }}, \phi\right] .
$$

Здесь мнимая единица возникает из квадратного корня из детерминанта метрики, который в форме Арновитта-Дезера-Мизнера имеет хорошо известный вид $g^{1 / 2}=N\left(\operatorname{det} g_{a b}\right)^{1 / 2}$. Заметим, что аналитическое продолжение от лоренцевой к 


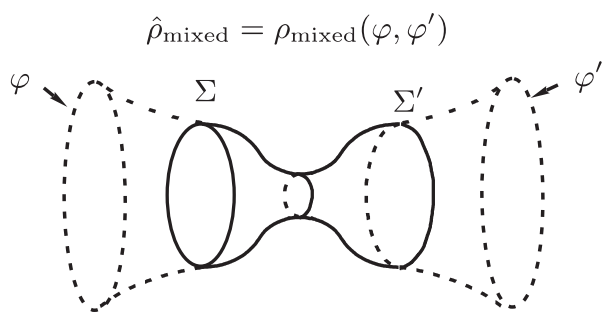

a
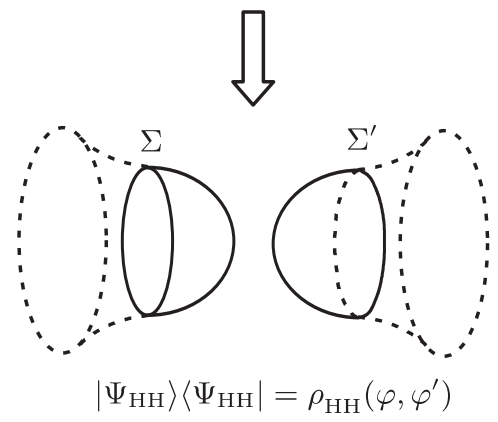

б

Рис. 2. Топология пространственно-временных конфигураций, лежащих в основе смешанных и чистых (типа Хартла-Хокинга) состояний в матрице плотности.

евклидовой картине происходит в комплексной плоскости функции хода, а не в комплексной плоскости времени (временна́я переменная одна и та же в обеих картинах), хотя, конечно, это эквивалентно обычному повороту Вика $t_{\text {Euclid }}=i t_{\text {Lorentz }}$.

В приведенных выше обозначениях матрица плотности (6) принимает вид интеграла по путям евклидовой квантовой гравитации:

$$
\rho\left(\varphi_{+}, \varphi_{-}\right)=e^{\Gamma} \int_{q\left(t_{ \pm}\right)=\varphi_{ \pm}} D\left[g_{\mu \nu}, \phi\right] e^{-S_{\mathrm{E}}\left[g_{\mu \nu}, \phi\right]} .
$$

Однако в силу равенства (7) область интегрирования евклидовой функции хода $N \equiv N_{\text {Euclid }}$ (в дальнейшем для краткости мы не будем писать нижний индекс) совпадает с мнимой осью,

$$
-i \infty<N<i \infty
$$

и в лагранжевой плотности евклидового действия выбор ветви квадратного корня из детерминанта метрики определяется как $g^{1 / 2}=N\left(\operatorname{det} g_{a b}\right)^{1 / 2}$. В дальнейшем эти соглашения будут играть очень важную роль.

Топология конфигураций, по которым ведется интегрирование в (8), представляет собой $\mathbb{R}^{1} \times S^{3}$, как это изображено на рис. 2 а. Топология пространственно-временного объема, интерполирующего между гиперповерхностями $\Sigma$ и $\Sigma^{\prime}$, отражает смешанный характер матрицы плотности и устанавливает перепутывающие корреляции между $\varphi$ и $\varphi^{\prime}$. Однако эти конфигурации включают как предельный случай несвязное пространство-время, получающееся путем пережимания и разрыва 


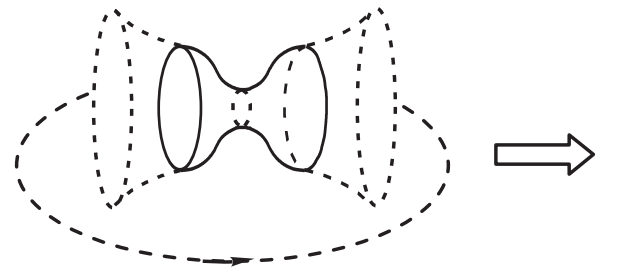

a
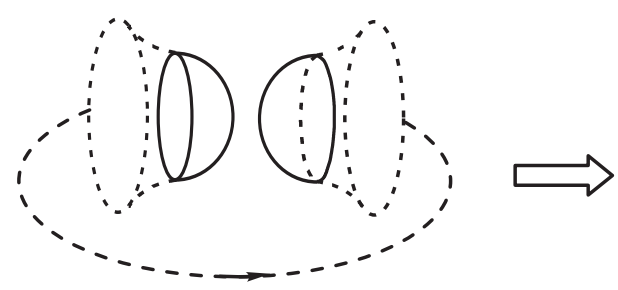

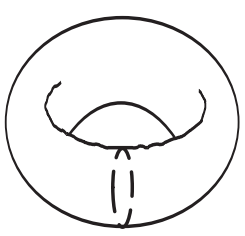

$\Sigma=\Sigma^{\prime}$

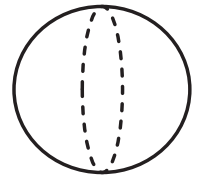

$\Sigma=\Sigma^{\prime}$

Рис. 3. Переход от матрицы плотности к ее статистической сумме для смешанных и чистых состояний.

пространственно-временного моста между $\Sigma$ и $\Sigma^{\prime}$ (см. рис. 2б). Оно ассоциируется со вкладом, который представляется как прямое произведение чистых состояний типа Хартла-Хокинга, показанных на рис. 1.

Нормировочный множитель $e^{\Gamma}$ в (8) вытекает из нормировки матрицы плотности $\operatorname{Tr} \hat{\rho}=1$ и определяет основной объект - статистическую сумму модели. Операция взятия следа подразумевает интегрирование по диагональным элементам матрицы плотности, так что статистическая сумма принимает вид интеграла по периодическим конфигурациям, пространственно-временная топология которых $\mathbb{R}^{1} \times S^{3}$ следует из определения краевых поверхностей $\Sigma$ и $\Sigma^{\prime}$,

$$
e^{-\Gamma}=\int_{\text {periodic }} D\left[g_{\mu \nu}, \phi\right] e^{-S_{\mathrm{E}}\left[g_{\mu \nu}, \phi\right]} .
$$

Для вкладов смешанных состояний это приводит к топологии $S^{1} \times S^{3}$, изображенной на рис. За, а вклад чистых состояний соответствует топологии $S^{4}$ (рис. 3б).

Дальнейшее вычисление статистической суммы может быть основано на выделении сектора минисуперпространства из полного конфигурационного пространства:

$$
\begin{gathered}
g_{\mu \nu}, \quad \phi \rightarrow a(\tau), N(\tau), \Phi(x), \quad \Phi(x)=\left(\phi(x), \psi(x), A_{\mu}(x), h_{\mu \nu}(x), \ldots\right) \\
d s^{2}=N^{2}(\tau) d \tau^{2}+a^{2}(\tau) d^{2} \Omega^{(3)}
\end{gathered}
$$

Тогда интеграл по путям может быть преобразован к виду интеграла по минисуперпространственной функции хода $N(\tau)$ и масштабному фактору $a(\tau)$ пространствен- 
но-замкнутой евклидовой метрики Фридмана-Робертсона-Уолкера (FRW):

$$
e^{-\Gamma}=\int D[a, N] e^{-S_{\mathrm{eff}}[a, N]},
$$

где

$$
e^{-S_{\mathrm{eff}}[a, N]}=\int D \Phi(x) e^{-S_{\mathrm{E}}[a, N ; \Phi(x)]} .
$$

Здесь $S_{\text {eff }}$ - евклидово эффективное действие неоднородных "материальных" полей $\Phi(x)=\Phi(\tau, \mathbf{x})$ (которые также включают в себя метрические возмущения $h_{\mu \nu}$ ) на минисуперпространственном фоне метрики FRW, а $S_{\mathrm{E}}[a, N ; \Phi(x)] \equiv S_{\mathrm{E}}\left[g_{\mu \nu}, \phi\right]$ - исходное евклидово действие, переписанное в терминах минисуперпространственного разложения (10).

Запись интеграла (12) в евклидовой форме удобна с точки зрения потребностей квазиклассического приближения. В этом приближении интеграл задается вкладом седловой точки, $\Gamma_{0}=S_{\text {eff }}\left[a_{0}, N_{0}\right]$, где $a_{0}=a_{0}(\tau)$ и $N_{0}=N_{0}(\tau)$ являются решениями уравнения движения для $S_{\text {eff }}[a, N]$,

$$
\frac{\delta S_{\mathrm{eff}}\left[a_{0}, N_{0}\right]}{\delta N_{0}(\tau)}=0,
$$

и удовлетворяют условию периодичности, вытекающему из определения статистической суммы. Такие периодические решения существуют при вещественном евклидовом значении функции $N$, а не в лоренцевой области с мнимой функцией хода. Это означает, что контур интегрирования по $N(9)$, совпадающий с мнимой осью, должен быть деформирован в контур на комплексной плоскости, чтобы пересекать вещественную ось при некотором $N_{0} \neq 0$, соответствующем евклидову решению уравнений движения для минисуперпространственного действия, как это изображено на рис. 4.

Остаточная инвариантность относительно одномерных диффеоморфизмов для этого действия (которая убирается процедурой фиксации калибровки, подразумеваемой в мере интегрирования $D[a, N])$ позволяет зафиксировать произвол в выбоpe $N_{0}$. В результате остается только свобода выбора положительной $\left(N_{0}>0\right)$ или отрицательной $\left(N_{0}<0\right)$ величины функции хода, поскольку, с одной стороны, все величины в каждом из этих двух классов калибровочно эквивалентны, с другой стороны, не существует непрерывного семейства невырожденных диффеоморфизмов, отображающих эти классы один в другой. Без потери общности можно выбрать в качестве представителей этих классов эквивалентности $N_{0}= \pm 1$ и обозначить решения и их действия на массовой оболочке соответственно как $a_{ \pm}(\tau)$ и

$$
\Gamma_{ \pm}=S_{\mathrm{eff}}\left[a_{ \pm}(\tau), \pm 1\right]
$$

Калибровочная неэквивалентность $\Gamma_{-} \neq \Gamma_{+}$этих двух классов очевидна, поскольку все вклады в эффективное действие являются нечетными функционалами от $N$, и $S_{\text {local }}[a, N]=-S_{\text {local }}[a,-N]$. Таким образом, можно сопоставить статистическую сумму $\Gamma_{+}$с хартл-хокинговским ("no-boundary") предписанием, a $\Gamma_{-}-$с туннелирующим предписанием для квантового состояния вселенной:

$$
e^{-\Gamma_{\text {no-boundary }}}=e^{-\Gamma_{+}}, \quad e^{-\Gamma_{\text {tunnel }}}=e^{-\Gamma_{-}} .
$$




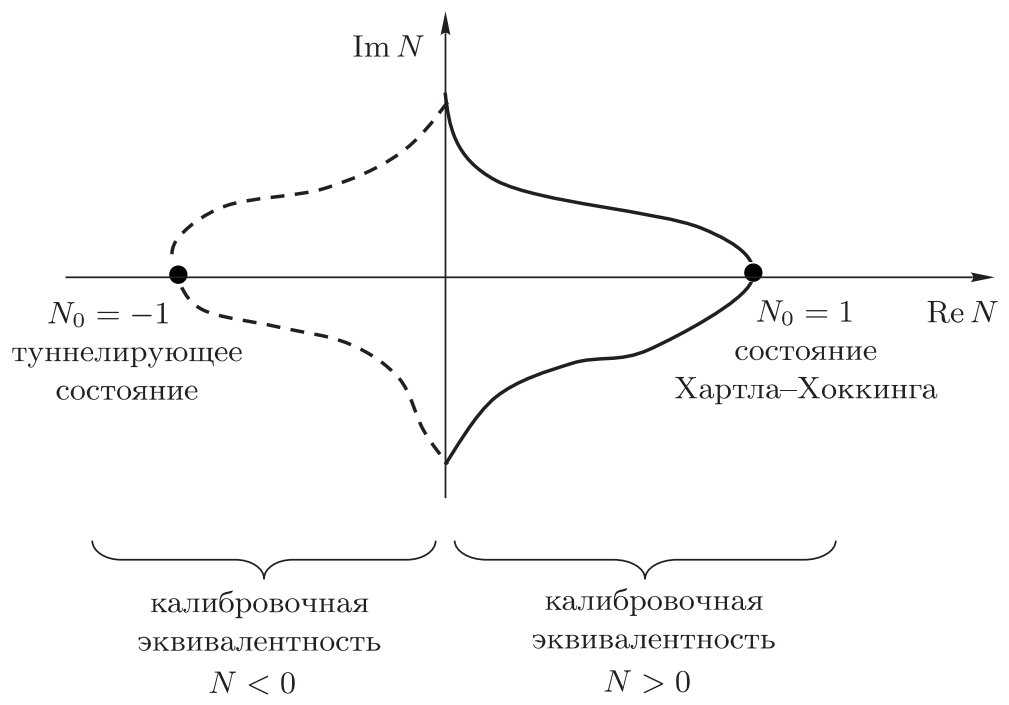

Рис. 4. Контуры интегрирования в комплексной плоскости, проходящие через хартл-хокинговскую ("no-boundary") и туннелирующую седловые точки интеграла.

Этот результат показывает, что для обоих предписаний полное квантовое эффективное действие как целое содержится в показателе экспоненты в функции распределения без какого-либо разделения на минисуперпространственный вклад и вклад материи, взятые с разными знаками, как в (2). Это означает, что обычная схема ренормировки применима к вычислению действия (15): общековариантные контрчлены должны быть получены на фоне метрики общего вида и затем вычислены в метрике FRW с $N= \pm 1$ в зависимости от выбора либо "no-boundary", либо туннелирующего предписания. Успех применения данного алгоритма определяется искусством вычисления эффективного действия $S_{\text {eff }}[a, N]$, которое можно найти в двух разных случаях - для безмассовых квантовых полей, конформно связанных с гравитацией, и в противоположном случае очень тяжелых массивных полей. В первом случае наличие локальной конформной симметрии и ее нарушение посредством точно рассчитываемой аномалии позволяет найти $S_{\text {eff }}[a, N]$ как функционал от общей метрики FRW $(a(\tau), N(\tau))$, в то время как во втором случае действие $S_{\text {eff }}[a, N]$ известно как ограничение на минисуперпространственный фон универсального разложения по обратным степеням массы (или разложения по степеням градиентов и кривизны пространства-времени).

\section{3. КОСМОЛОГИЯ, ГЕНЕРИРУЕМАЯ КОНФОРМНОЙ ТЕОРИЕЙ ПОЛЯ: НОВЫЙ СТАТУС СОСТОЯНИЯ ХАРТЛА-ХОКИНГА}

В настоящем разделе мы приводим приложение уравнений (14)-(16) к построению состояния Хартла-Хокинга в гравитационной теории с материальным сектором, в котором доминируют свободные (линейные) поля, конформно взаимодействующие 
с гравитацией (CFT):

$$
S_{\mathrm{E}}\left[g_{\mu \nu}, \phi\right]=-\frac{1}{16 \pi G} \int d^{4} x g^{1 / 2}(R-2 \Lambda)+S_{\mathrm{CFT}}\left[g_{\mu \nu}, \phi\right] .
$$

Эффективное действие в такой системе в основном исчерпывается квантовым действием этих конформных полей, которые просто превосходят по численности неконформные поля (включая гравитон). В свою очередь, это действие может быть точно вычислено путем конформного преобразования, превращающего метрику (11) в статическую эйнштейновскую метрику с $a=$ const. В единицах массы Планка $m_{\mathrm{P}}=(3 \pi / 4 G)^{1 / 2}$ действие имеет вид [15]

$$
S_{\mathrm{eff}}[a, N]=m_{\mathrm{P}}^{2} \oint d \tau N\left\{-a\left(a^{\prime}\right)^{2}-a+\frac{\Lambda}{3} a^{3}+B\left(\frac{\left(a^{\prime}\right)^{2}}{a}-\frac{\left(a^{\prime}\right)^{4}}{6 a}\right)+\frac{B}{2 a}\right\}+F(\eta),
$$

где

$$
F(\eta)= \pm \sum_{\omega} \ln \left(1 \mp e^{-\omega \eta}\right), \quad \eta=\oint \frac{d \tau N}{a}, \quad a^{\prime} \equiv \frac{d a}{N d \tau} .
$$

Первые три члена в фигурных скобках в правой части (17) представляют эйнштейновское действие с первичной космологической постоянной $\Lambda \equiv 3 H^{2}(H$ - соответствующая постоянная Хаббла), первый из членов, пропорциональных $B$, отвечает вкладу конформной аномалии, а второй $(B / 2 a)$ - вкладу вакуумной энергии (Казимира) конформных полей на статическом пространстве Эйнштейна, $F(\eta)$ является свободной энергией этих полей, т. е. типичной бозонной или фермионной суммой по полевым осцилляторам с энергиями $\omega$ на единичной 3-сфере, при этом $\eta$ играет роль обратной температуры (полный период инстантона в единицах конформного времени). Константа $B=3 \beta / 4 m_{\mathrm{P}}^{2}$ определяется коэффициентом $\beta$ при топологическом инварианте Гаусса-Бонне $E=R_{\mu \nu \alpha \gamma}^{2}-4 R_{\mu \nu}^{2}+R^{2}$ в полной конформной аномалии квантовых полей:

$$
\begin{gathered}
g_{\mu \nu} \frac{\delta S_{\mathrm{eff}}^{\mathrm{CFT}}}{\delta g_{\mu \nu}}=\frac{1}{4(4 \pi)^{2}} g^{1 / 2}\left(\alpha \square R+\beta E+\gamma C_{\mu \nu \alpha \beta}^{2}\right), \\
e^{-S_{\mathrm{eff}}^{\mathrm{CFT}}\left[g_{\mu \nu}\right]}=\int D[\phi] e^{-S_{\mathrm{CFT}}\left[g_{\mu \nu}, \phi\right]} .
\end{gathered}
$$

Здесь $S_{\text {eff }}^{\mathrm{CFT}}\left[g_{\mu \nu}\right]$ - эффективное действие этих конформных полей во внешнем гравитационном поле и $C_{\mu \nu \alpha \beta}^{2}-$ квадрат тензора Вейля.

Как показано в работах [15], [16], [32], решения эффективных уравнений (14) в этой модели порождают набор периодических инстантонов типа гирлянд с осциллирующим масштабным фактором и топологией $S^{1} \times S^{3}$ (которые можно рассматривать как термическую версию инстантонов Хартла-Хокинга), а также вакуумные инстантоны Хартла-Хокинга с топологией $S^{4}$ (см. рис. 5). Эффективное уравнение Фридмана (14)

$$
-\frac{\left(a^{\prime}\right)^{2}}{a^{2}}+\frac{1}{a^{2}}-B\left(\frac{\left(a^{\prime}\right)^{4}}{2 a^{4}}-\frac{\left(a^{\prime}\right)^{2}}{a^{4}}\right)=\frac{\Lambda}{3}+\frac{C}{a^{4}}
$$

где

$$
C=\frac{B}{2}+\frac{1}{m_{\mathrm{P}}^{2}} \frac{d F(\eta)}{d \eta}=\frac{B}{2}+\frac{1}{m_{\mathrm{P}}^{2}} \sum_{\omega} \frac{\omega}{e^{\omega \eta} \mp 1}, \quad \eta=\oint \frac{d \tau}{a}
$$




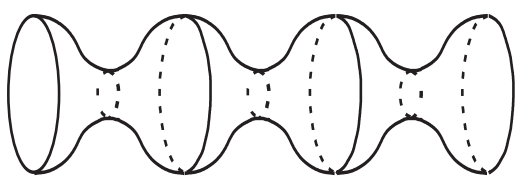

гирлянда

с $k$ осцилляциями

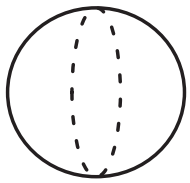

инстантон $S^{4}$

Рис. 5. Периодические инстантоны типа гирлянд с осциллирующим масштабным фактором (предполагается, что правая и левая границы гирлянды с $k=1,2, \ldots$ осцилляциями отождествлены) и инстантон Хартла-Хокинга $S^{4}$.

отличается от приведенного выше аномальным членом, пропорциональным $B$, и радиационным членом $C / a^{4}$ с константой $C$, характеризующей сумму энергии Казимира и энергии газа термически возбужденных частиц с обратной температурой $\eta$ (напомним, $\eta$ - период инстантона в единицах конформного времени). Величина $\eta$ задается в (19) в виде интеграла по полному периоду $\tau$ или $2 k$-кратного интеграла между точками поворота $\tau_{ \pm}$масштабного фактора $a(\tau), \dot{a}\left(\tau_{ \pm}\right)=0$. Эта $k$-кратная природа интеграла подразумевает, что в периодическом решении масштабный фактор осциллирует $k$ раз между максимальным и минимальным значениями $a_{ \pm}=a\left(\tau_{ \pm}\right)$, $a_{-} \leqslant a(\tau) \leqslant a_{+}$, где

$$
a_{ \pm}^{2}=\frac{1}{2 H^{2}}\left(1 \pm \sqrt{1-4 H^{2} C}\right) .
$$

Таким образом, период решений определяется как функция от $G$ и $\Lambda$ из второго из уравнений (19) и не может быть задан произвольным образом. Это является особенностью микроканонического ансамбля [16] с только двумя фиксируемыми константами - перенормированной гравитационной и космологической постоянными.

Как показано в работе [15], инстантоны с топологией $S^{3} \times S^{1}$ существуют только в ограниченной области космологической постоянной $\Lambda$, а именно при

$$
0<\Lambda_{\min }<\Lambda<\Lambda_{\max }=\frac{3}{2 B}
$$

В этой области они образуют последовательность однопараметрических семейств, изображенных на рис. 6 , в плоскости $(\Lambda, C)$, где $\Lambda$ - космологическая постоянная, а $C$ - константа, определяющая количество радиации в системе (включая наряду с энергией теплового газа, см. (19), также энергию Казимира B/2). Эти семейства интерполируют между двумя границами кривоугольного треугольника в области инстантонов на плоскости $(\Lambda, C)$ - между прямой линией нижней границы $C=B-B^{2} \Lambda / 3$ и верхней гиперболической границей $C=3 / 4 \Lambda$. В силу этого спектр допустимых значений $\Lambda$ внутри интервала (21) имеет зонную структуру: зоны $\Delta_{k}$, $k=1,2, \ldots$, образуются при проекции этих семейств на ось $\Lambda$. Их последовательность при $k \rightarrow \infty$ сгущается в углу этого треугольника - на верхней границе области (21). Эта граница представляет собой новый масштаб квантовой гравитации, который стремится к бесконечности, когда выключаются квантовые эффекты, т. е. при $\beta \rightarrow 0$. Нижняя граница $\Lambda_{\min }$ есть низшая точка семейства с $k=1$, она может быть 


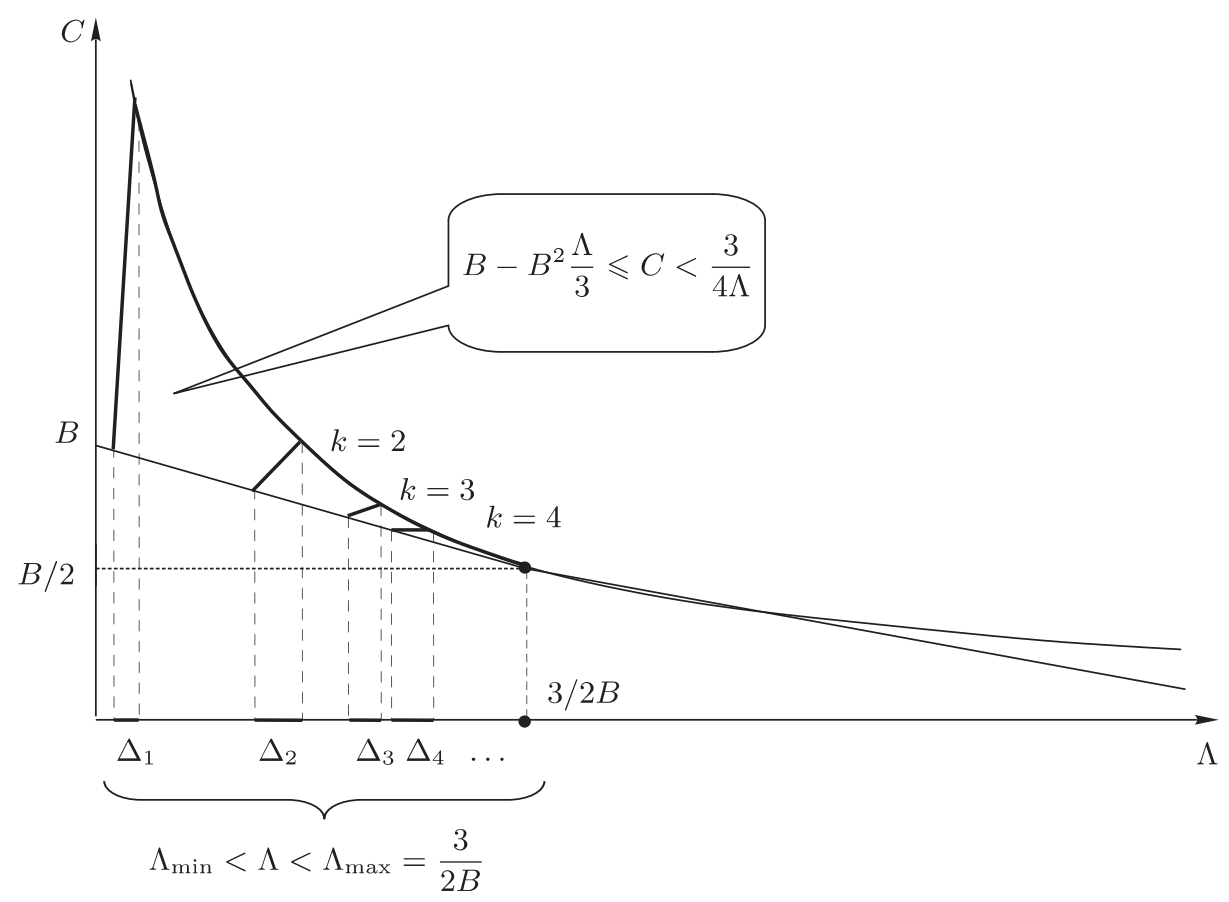

Рис. 6. Зонная структура спектра космологической постоянной для термических состояний в космологии СFT.

численно рассчитана для любого полевого содержания модели. При большом количестве конформных полей, а следовательно, при большом $\beta$ порядка $N$, нижняя граница имеет порядок $1 / \beta G$. Таким образом, ограничение (21) предлагает своего рода $1 / N$-решение проблемы космологической постоянной, поскольку, задавая достаточно большое число конформных полей, можно получить начальное значение $\Lambda$ существенно ниже масштаба Планка, где применима эффективная теория, но достаточное для генерации длинной инфляционной стадии. Также это ограничение можно потенциально рассматривать как критерий выбора в ландшафте струнных вакуумов [15], [16].

Решение системы (18), (19) также включает в себя вакуумные инстантоны ХартлаХокинга без радиации, $d F / d \eta=0$. Они представляют евклидово пространствовремя де Ситтера с эффективным фактором Хаббла

$$
H_{\mathrm{eff}}^{2}=\frac{1-\sqrt{1-2 B H^{2}}}{B}
$$

соответствующим вырожденному случаю пространства $S^{3} \times S^{1}$, разорванного при нулевом значении масштабного фактора $a_{-}=0$ и топологически принявшего вид 4-сферы $S^{4}$, которая описывает чисто вакуумный вклад в статистическую сумму. Вакуумная природа этих инстантонов следует из того факта, что их период конформного времени в (19) расходится при $\tau_{-}$в силу $a_{-}=0$ и порождает нулевую эффективную температуру порядка $1 / \eta$ с $F(\eta)=0$. Такие решения существуют для 
всех $\Lambda \leqslant 3 / 2 B$ (горизонтальный сегмент при $C=B / 2$ на рис. 6 ), но они выпадают из статистической суммы в силу их бесконечного положительного эффективного действия. Это свойство следует из вклада конформной аномалии (см. зависимость типа $1 / a$ у кинетического члена, пропорционального $B$, в эффективном действии $(17))^{2)}$. Следовательно, предсказания древесного приближения теории с отрицательным евклидовым действием изменяются критическим образом из-за эффекта конформной аномалии.

3.1. Туннелирующее состояние. Ситуация с туннелирующим состоянием в космологии CFT существенно сложнее. Начнем с того, что попытка вычислить эффективное действие для $N>0$, а затем непосредственно аналитически продолжить результат к отрицательным $N$ с $\eta<0$ не удается, потому что эти две области разделены мнимой осью, плотно заполненной точками ветвления логарифма в функции $F(\eta)$ при $\omega \eta=(2 n+1) i \pi$ для всех целых $n$ и всех дискретных значений $\omega$ спектра полевых осцилляторов ${ }^{3)}$.

Альтернативный подход мог бы состоять в прямом вычислении и перенормировке эффективного действия с отрицательными $N$ и $\eta$, т. е. в замене $\eta \mapsto-\eta$ в (17) до перенормировки. Заметим, что аномальная часть действия (17)

$$
S_{\text {anomaly }}[a, N]=m_{\mathrm{P}}^{2} B \oint d \tau N\left(\frac{\left(a^{\prime}\right)^{2}}{a}-\frac{\left(a^{\prime}\right)^{4}}{6 a}\right)
$$

и его конечная часть $\eta m_{\mathrm{P}}^{2} B / 2$, связанная с энергией Казимира, обусловлены ковариантной регуляризацией четвертичных, квадратичных и логарифмических расходимостей в части эффективного действия, формально задаваемой его вакуумной энергией $\eta \sum_{\omega}(\omega / 2)$. Это свойство может быть записано как соотношение

$$
\left[\eta \sum_{\omega} \frac{\omega}{2}\right]^{\text {renorm }}=\frac{m_{\mathrm{P}}^{2} B}{2} \eta+S_{\text {anomaly }}[a, N],
$$

означающее, что аномальная часть действия возникает как своего рода "хвост" к вычитанию ультрафиолетовых расходимостей в $\eta \sum_{\omega}(\omega / 2)$ и, следовательно, строго коррелирована с последними.

Наивно рассуждая, можно подумать, что инверсия $\eta \mapsto-\eta$ в (18) обратит знак аномальной части и, таким образом, бесконечно усилит вклад инстантона $S^{4}$ в туннелирующем случае (в отличие от ситуации Хартла-Хокинга). Это умозаключение, однако, неправильное, потому что после инверсии $\eta \mapsto-\eta$ свободная энергия $F(-\eta)$ при $\eta>0$ также становится ультрафиолетово-расходящейся, $\left.F(-\eta)\right|^{\mathrm{div}}=\left.\eta \sum_{\omega} \omega\right|^{\mathrm{div}}$, следовательно, требуется дополнительный контрчлен, который фактически восстанавливает тот же самый (хартл-хокинговский) знак аномального члена. Чтобы это увидеть, сделаем цепочку простых преобразований (справедливых по модулю мнимой не зависящей от полей части) в квантовой части туннелирующего эффективного

2) Заметим, что на вакуумном решении уравнения $(18)\left(a^{\prime}\right)^{2}\left(\tau_{-}\right)=1$, и подынтегральное выражение в (17) стремится к $+\infty$ в точке $\tau_{-}$, при этом $a \rightarrow 0$.

3) Ультрафиолетовая регуляризация посредством обрезания на максимальном значении $\omega$ открывает маленький мостик в окрестности нуля между полуплоскостями комплексного $N$, но результат аналитического продолжения через такую область далеко не очевиден. 
действия:

$$
\begin{aligned}
{\left[-\eta \sum_{\omega} \frac{\omega}{2}+F(-\eta)\right]^{\text {renorm }} } & =\left[\eta \sum_{\omega} \frac{\omega}{2}+F(\eta)\right]^{\text {renorm }}= \\
& =S_{\text {anomaly }}[a, N]+\frac{m_{\mathrm{P}}^{2} B}{2} \eta+F(\eta), \quad \eta, N>0 .
\end{aligned}
$$

Эти преобразования означают, что только классическая эйнштейновская часть полного действия получает противоположный знак, в то время как квантовая часть остается такой же, как и в случае состоянии Хартла-Хокинга. Эффективно это соответствует тому, что в уравнениях движения (18), (19) нужно поменять знак только у константы $C$. Замена $C \mapsto-C$ подразумевает в силу $(20)$, что $a_{-}^{2}<0$, таким образом, масштабный фактор евклидового решения изменяется от $a=0$ до $a_{+}$и соответствует вакуумному инстантону $S^{4}$ Хартла-Хокинга ${ }^{4)}$. Однако в силу (24) полное эффективное действие стремится к $+\infty$, как и в случае состояния Хартла-Хокинга, и исключает туннелирующий вклад. В результате мы приходим к выводу, что в космологии СFT туннелирующее состояние динамически запрещено.

\section{4. ТЯЖЕЛЫЕ МАССИВНЫЕ ПОЛЯ: СОСТОЯНИЕ ХАРТЛА-ХОКИНГА И ТУННЕЛИРУЮЩЕЕ СОСТОЯНИЕ}

В случае тяжелых массивных квантовых полей ситуация более благоприятна для туннелирующего состояния. В настоящем разделе мы рассмотрим состояние Хартла-Хокинга и туннелирующее состояние одновременно, поскольку их формализмы очень похожи. Теперь евклидово эффективное действие имеет универсальный вид градиентного разложения по обратным степеням массового параметра:

$$
S_{\mathrm{eff}}\left[g_{\mu \nu}\right]=\int d^{4} x g^{1 / 2}\left(M_{\mathrm{P}}^{2} \Lambda-\frac{M_{\mathrm{P}}^{2}}{2} R\left(g_{\mu \nu}\right)+\cdots\right),
$$

где мы отбрасываем члены более высоких степеней по кривизне и производным среднего значения материальных полей. Мы можем рассматривать космологический член и квадрат (редуцированной) массы Планка $M_{\mathrm{P}}^{2}=1 / 8 \pi G$ как функции от этих средних и считать их константами в приближении медленно меняющихся полей. Эффективное действие (25) не содержит тепловой части, характерной для статистического ансамбля [15], потому что для тяжелых полей радиация не возбуждается. Это объясняется тем, что эффективная температура оказывается исчезающе малой.

Фактически функционал минисуперпространственного действия для (25) выглядит в единицах $m_{\mathrm{P}}^{2}=3 \pi / 4 G=6 \pi^{2} M_{\mathrm{P}}^{2}$ как

$$
S_{\mathrm{eff}}[a, N]=m_{\mathrm{P}}^{2} \int d \tau N\left(-a\left(a^{\prime}\right)^{2}-a+H^{2} a^{3}\right),
$$

где мы используем обозначение для космологической постоянной $\Lambda=3 H^{2}$ в терминах эффективного фактора Хаббла $H$. Тогда седловая точка для интеграла по

\footnotetext{
4) Его период конформного времени $\eta$ расходится, так что $F(\eta)=0$ и $C=0$, а уравнения движения дают точное деситтеровское решение с эффективной постоянной Хаббла (22).
} 
путям (12) - точка стационарности по отношению к вариациям функции хода, определяющаяся условием $\delta S_{\text {eff }}[a, N] / \delta N=0$, - удовлетворяет евклидову уравнению Фридмана

$$
\left(a^{\prime}\right)^{2}=1-H^{2} a^{2} .
$$

Оно имеет точку поворота при $a_{+}=1 / H$, ниже которой вещественное решение интерполирует между $a_{-} \equiv a(0)=0$ и $a_{+}$. В калибровке $N= \pm 1$ для обоих состояний это решение описывает евклидову метрику де Ситтера, т. е. одну полусферу $S^{4}$ (изображенную на рис. 1),

$$
a_{ \pm}(\tau)=\frac{1}{H} \sin (H \tau) .
$$

После отскока траектории масштабного фактора $a(\tau)$ от его максимального значения $a_{+}$, соответствующего экваториальному сечению 4-сферы, это решение описывает на стадии сжатия остальную часть этой сферы. Таким образом, оно не является периодическим и согласно сказанному выше описывает чисто вакуумный вклад в статистическую сумму (12). Аналогично случаю конформных полей эффективная температура этого состояния определяется обратным полным периодом $\eta$. Следовательно, для решения (28) она обращается в нуль, потому что этот период между полюсами данного сферического инстантона стремится к бесконечности. Это оправдывает отсутствие тепловой части в (25).

Итак, при $N= \pm 1$ действие Хартла-Хокинга и туннелирующее действие (15) имеют вид

$$
\Gamma_{ \pm}=\mp \frac{8 \pi^{2} M_{\mathrm{P}}^{2}}{H^{2}}
$$

и главный предмет нашего интереса - туннелирующая функция распределения в пространстве положительных значений $H^{2}=\Lambda / 3-$ равна

$$
\rho_{\text {tunnel }}(\Lambda)=\exp \left(-\frac{24 \pi^{2} M_{\mathrm{P}}^{2}}{\Lambda}\right), \quad \Lambda>0 .
$$

Она совпадает с квазиклассической туннелирующей волновой функцией вселенной [2] $\left|\Psi_{\text {tunnel }}\right|^{2} \simeq \exp \left(-8 \pi^{2} M_{\mathrm{P}}^{2} / H^{2}\right)$, выведенной из уравнения Уилера-де Витта в древесном приближении теории.

В точке поворота $a_{+}$решение (28) может быть аналитически продолжено в лоренцев режим, $a_{\mathrm{L}}(t)=a(\pi / 2 H+i t)$. Тогда эволюция масштабного фактора описывается уравнением

$$
a_{\mathrm{L}}(t)=\frac{1}{H} \operatorname{ch}(H t)
$$

что можно интерпретировать как распределение масштабных факторов в квантовом ансамбле (после декогерентизации) деситтеровских моделей, распределенных в соответствии с (29) (см. рис. 1). Заметим, что попытка расширить этот ансамбль на отрицательные значения $\Lambda$ не удается, потому что уравнение (27) при $H^{2}<0$ не имеет точек поворота с отщепляющимся вещественным лоренцевым пространством-временем. Более того, виртуальные космологические модели с евклидовой сигнатурой также запрещены в туннелирующем состоянии, ввиду того что их положительное евклидово действие стремится к бесконечности, в результате $\rho_{\text {tunnel }}(\Lambda)=0$ для $\Lambda<0$. 


\section{5. КВАНТОВОЕ ПРОИСХОЖДЕНИЕ ВСЕЛЕННОЙ С ИНФЛАТОНОМ ХИГГСА СТАНДАРТНОЙ МОДЕЛИ}

Функция распределения вышеуказанного типа может служить в качестве источника начальных условий для инфляции только в том случае, когда космологическая постоянная $\Lambda=3 H^{2}$ становится композитным полем, которое может распадаться при выходе из режима инфляции. Обычно оно представляет собой скалярное поле инфлатона, квантовое среднее которого $\varphi$ почти постоянно в режиме медленного скатывания, а его эффективный потенциал $V(\varphi)$ играет роль космологической постоянной, генерирующей инфляцию.

Когда вклад градиентов инфлатона мал, рассматриваемый формализм можно применять также с учетом этого поля, окончательный эффект которого сводится к генерированию космологической постоянной $\Lambda=V(\varphi) / M_{\mathrm{P}}^{2}$ и эффективной массы Планка. Эти константы являются коэффициентами нулевого и первого порядка разложения эффективного действия по степеням кривизны и включают в себя радиационные поправки от всех квантовых полей в интеграле по путям (13). Теперь расхождение в знаках древесной и петлевой частей функции распределения отсутствует, следовательно, можно применить стандартную схему перенормировок и, если необходимо, РГ-улучшение, чтобы получить полное эффективное действие $S_{\text {eff }}\left[g_{\mu \nu}, \varphi\right]$ и затем повторить процедуру, описанную в предыдущем разделе. В приближении медленного скатывания эффективное действие имеет общий вид

$$
S_{\mathrm{eff}}\left[g_{\mu \nu}, \varphi\right]=\int d^{4} x g^{1 / 2}\left(V(\varphi)-U(\varphi) R\left(g_{\mu \nu}\right)+\frac{1}{2} G(\varphi)(\nabla \varphi)^{2}+\cdots\right),
$$

где $V(\varphi), U(\varphi)$ и $G(\varphi)$ представляют собой коэффициенты разложения по производным и отброшен вклад операторов с высшими производными. В случае медленно меняющегося инфлатона коэффициенты $V(\varphi)$ и $U(\varphi)$ играют роль эффективной космологической константы и квадрата массы Планка, так что в (25) и (26) можно отождествить $M_{\mathrm{P}}^{2}=m_{\mathrm{P}}^{2} / 6 \pi^{2}$ и $H^{2}$ соответственно с $2 U(\varphi)$ и $V(\varphi) / 6 U(\varphi)$. Таким образом, туннелирующая функция распределения (29) приобретает вид следующего распределения поля $\varphi$ :

$$
\rho_{\text {tunnel }}(\varphi)=\exp \left(-\frac{24 \pi^{2} M_{\mathrm{P}}^{4}}{\widehat{V}(\varphi)}\right)
$$

где

$$
\widehat{V}(\varphi)=\left(\frac{M_{\mathrm{P}}^{2}}{2}\right)^{2} \frac{V(\varphi)}{U^{2}(\varphi)}
$$

фактически совпадает [29], [30] с потенциалом в эйнштейновской параметризации действия (31).

Теперь мы применим этот формализм к модели (4) инфляции, генерируемой инфлатоном Хиггса СМ $\varphi=\left(\Phi^{\dagger} \Phi\right)^{1 / 2}$. Как показано в работах [29], [30], однопетлевое действие, улучшенное путем РГ-пересуммирования для больших $\varphi$, имеет вид (31) с коэффициентными функциями

$$
V(\varphi)=\frac{\lambda(t)}{4} Z^{4}(t) \varphi^{4}, \quad U(\varphi)=\frac{1}{2}\left(M_{\mathrm{P}}^{2}+\xi(t) Z^{2}(t) \varphi^{2}\right), \quad G(\varphi)=Z^{2}(t),
$$


определенными в терминах бегущих констант связи $\lambda(t)$ и $\xi(t)$ и перенормировки поля $Z(t)$. Они включают в себя эффект суммирования степеней логарифмов и принадлежат к решениям РГ-уравнений, которые на инфляционной стадии с большим $\varphi$ порядка $M_{\mathrm{P}} / \sqrt{\xi}$ и большим $\xi \gg 1$ выглядят как (см. подробности в [29], [30])

$$
\frac{d \lambda}{d t}=\frac{\mathbf{A}}{16 \pi^{2}} \lambda-4 \gamma \lambda, \quad \frac{d \xi}{d t}=\frac{6 \lambda}{16 \pi^{2}} \xi-2 \gamma \xi
$$

где $d Z / d t=\gamma Z$. Здесь $\gamma-$ аномальная размерность поля Хиггса, бегущий масштаб $t=\ln \left(\varphi / M_{\mathrm{t}}\right)$ нормирован на массу топ-кварка $\mu=M_{\mathrm{t}}$, а $\mathbf{A}=\mathbf{A}(t)$ - бегущий параметр аномального скейлинга. Эта величина была введена в работе [10] как коэффициент перед логарифмом эффективного потенциала всех физических частиц СМ и голдстоуновских мод. Благодаря четвертичным и калибровочным взаимодействиям и взаимодействию Юкавы с полем инфлантона $\varphi$ они приобретают массы $m(\varphi) \sim \varphi$ и при больших $\varphi$ приводят к асимптотике потенциала Коулмена-Вайнберга

$$
V^{1 \text {-loop }}(\varphi)=\sum_{\text {particles }}( \pm 1) \frac{m^{4}(\varphi)}{64 \pi^{2}} \ln \frac{m^{2}(\varphi)}{\mu^{2}} \simeq \frac{\lambda \mathbf{A}}{128 \pi^{2}} \varphi^{4} \ln \frac{\varphi^{2}}{\mu^{2}},
$$

с помощью которой можно определить А. Важность этого объекта и его модификации за счет РГ-бега константы неминимального взаимодействия $\xi(t)$,

$$
\mathbf{A}_{\mathrm{I}}=\mathbf{A}-12 \lambda
$$

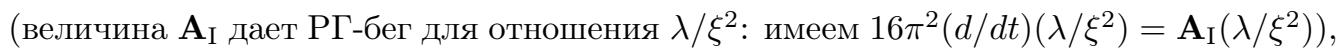
в том, что при $\xi \gg 1$ эта величина определяет квантовую инфляционную динамику [14], [33] и параметры генерируемого на стадии инфляции СМВ [24]. В частности, величина поля $\varphi$ в начале инфляционной стадии с продолжительностью $N$ в единицах показателя коэффициента экспоненциального расширения оказывается равной [24]

$$
\varphi^{2}=-\frac{64 \pi^{2} M_{\mathrm{P}}^{2}}{\xi \mathbf{A}_{\mathrm{I}}\left(t_{\mathrm{end}}\right)}\left(1-e^{x}\right),
$$

где параметр $x \equiv N \mathbf{A}_{\mathrm{I}}\left(t_{\mathrm{end}}\right) / 48 \pi^{2}$ непосредственно зависит от значения величины $\mathbf{A}_{\mathrm{I}}\left(t_{\text {end }}\right)$, взятой в конце инфляции, в момент времени $t_{\text {end }}=\ln \left(\varphi_{\text {end }} / M_{\mathrm{t}}\right)$, где $\varphi_{\text {end }} \simeq 2 M_{\mathrm{P}} / \sqrt{3 \xi}$. Этот параметр также входит в известный алгоритм нахождения спектра $\mathrm{CMB} \Delta_{\zeta}^{2}(k)$, и его спектральный индекс равен $n_{s}(k)$. Как показано в работах [29], [30], применение этого алгоритма с учетом экспериментальных ограничений $\Delta_{\zeta}^{2}\left(k_{0}\right) \simeq 2.5 \cdot 10^{-9}$ и $0.94<n_{s}\left(k_{0}\right)<0.99$ (данные космического зонда WMAP, данные о барионных осцилляциях и сверхновых в базовой точке $k_{0}=0.002 \mathrm{M}^{-1}$, соответствующей $N \simeq 60$ [34]) дает совместную с данными наблюдений СМВ область значений массы бозона Хиггса 135.6 ГэВ $\lesssim M_{\mathrm{H}} \lesssim 184.5$ ГэВ, причем обе границы этой области определяются нижним пределом спектрального индекса.

Теперь мы хотим показать, что в дополнение к хорошему соответствию спектра космологических возмущений с данными наблюдений СМВ, эта модель может также описывать механизм генерирования самого́ космологического фона, на котором эти возмущения существуют. Этот механизм состоит в образовании начальных условий для инфляции в форме острого вероятностного пика в функции распределения (32) при некотором значении поля инфлатона $\varphi_{0}$, при котором вселенная 


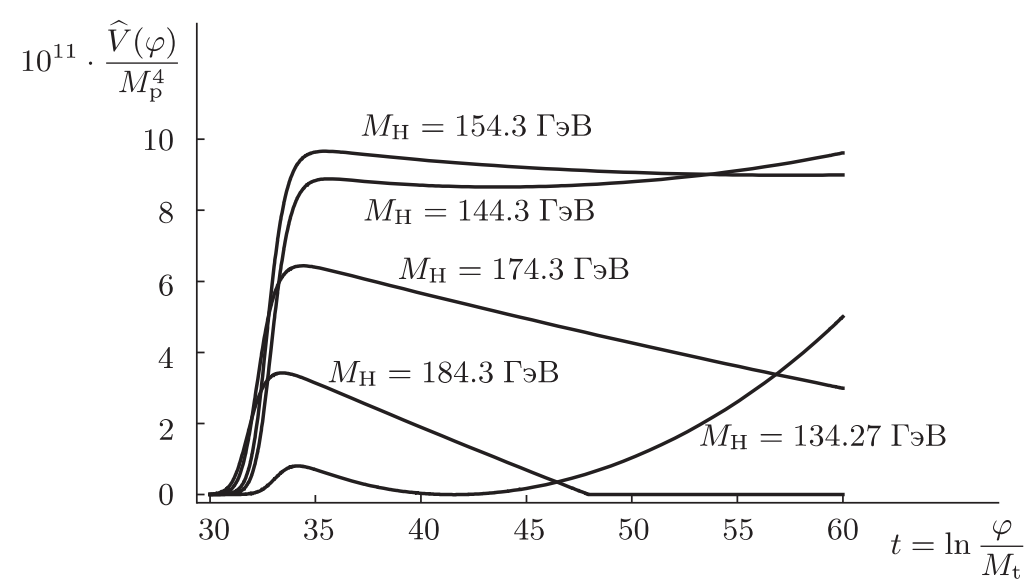

Рис. 7. Графики эффективного потенциала при разных значениях порога нестабильности EW-вакуума от $M_{\mathrm{H}}=M_{\mathrm{H}}^{\mathrm{inst}}=134.27$ ГэВ до $M_{\mathrm{H}}=184.3$ ГэВ, показывающие процесс возникновения метастабильного вакуума, за которым при больших $M_{\mathrm{H}}$ следует формирование семейства потенциалов с отрицательным склоном. Локальные пики потенциала $\widehat{V}$, расположенные при $t=34 \div 35$, растут вместе с $M_{\mathrm{H}}$ для $M_{\mathrm{H}} \lesssim 160$ ГэВ и начинают уменьшаться при больших $M_{\mathrm{H}}[29]$.

как целое начинает свою эволюцию [20]. Форма потенциала и его величина (33), изображенные на рис. 7 для нескольких значений массы бозона Хиггса, ясно демонстрируют существование этого пика. Действительно, взятая с обратным знаком обратная величина потенциала после экспоненцирования подавляет до нуля вероятность тех значений $\varphi$, в которых $\widehat{V}(\varphi) \rightarrow+0$, и, наоборот, увеличивает вероятность реализации положительных максимумов потенциала. Пример такого поведения при возрастании значения массы $M_{\mathrm{H}}$ следующий.

Как известно, для малых $M_{\mathrm{H}} \mathrm{CM}$ имеет область нестабильного EW-вакуума, характеризуемую отрицательными значениями $\lambda(t)$ на некоторых энергетических масштабах. Поэтому мы начнем рассмотрение с порога EW-нестабильности [35], [36], который существует в нашей гравитирующей $\mathrm{CM}$ при $M_{\mathrm{H}}^{\text {inst }} \approx 134.27$ ГэВ [29], [30] и который лежит немного ниже, чем область значений массы бозона Хиггса, согласующейся с данными СMB (значение $M_{\mathrm{H}}^{\text {inst }}$ использовано для кривой на рис. 8 и для нижней из кривых на рис. 7$)$. Потенциал $\widehat{V}(\varphi)$ падает до нуля при $t_{\text {inst }} \simeq 41.6$, $\varphi_{\text {inst }} \sim 80 M_{\mathrm{P}}$ и образует ложный вакуум [29], [30], отделенный от EW-вакуума высоким пиком при $t \simeq 34$. Таким образом, вероятность образования вселенной с начальным значением инфлатона на $\mathrm{EW}$-масштабе $\varphi=v$ и на масштабе $\varphi_{\text {inst }}$ подавляется до нуля, в то время как наиболее вероятное значение принадлежит области локализации этого пика. Инфляционная стадия формирования базового возмущения СMB, соответствующего $N=60$ (от момента $t_{\text {in }}$ пересечения горизонта до конца инфляции $\left.t_{\text {end }}\right)$, которая отмечена штриховыми линиями на рис. 8 , лежит слева от этого пика. Это отвечает требованию хронологической последовательности начальных условий для инфляции и формирования спектра СМВ. 


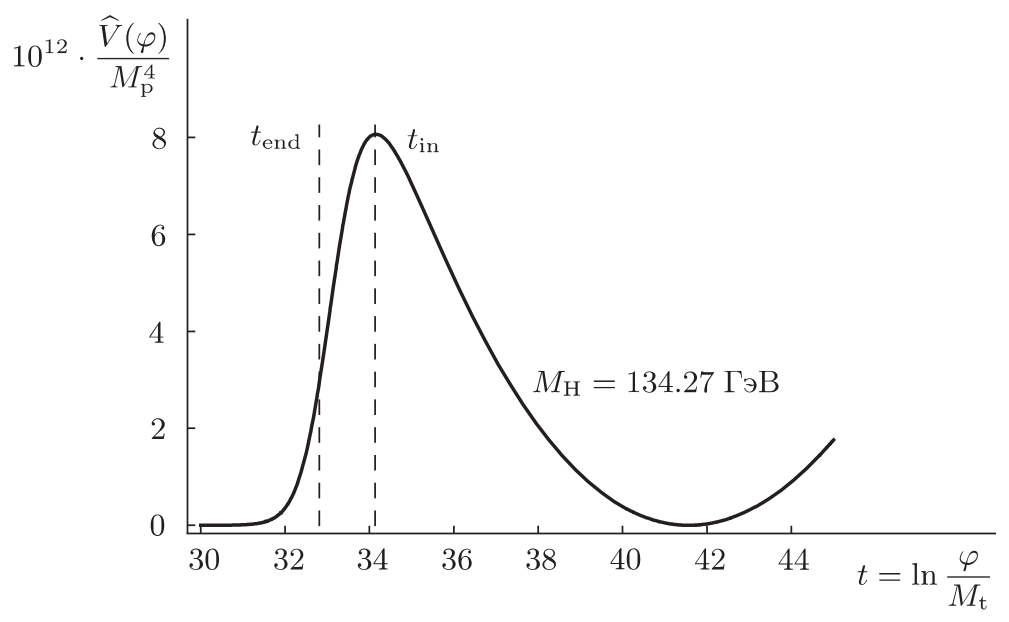

Рис. 8. Эффективный потенциал для порога нестабильности $M_{\mathrm{H}}^{\text {inst }}=$ 134.27 ГэВ. Ложный вакуум имеет место при $t_{\text {inst }} \simeq 41.6, \varphi \sim 80 M_{\text {P. Область }}$ инфляции для $N=60$ возмущения СМВ (определяемая границей, полученной по данным WMAP) обозначена штриховыми линиями [29].

Описанный случай, однако, лежит ниже совместной с данными СМВ областью $M_{\mathrm{H}}$ и был рассмотрен только с целью иллюстрации ${ }^{5)}$. Представляющее интерес поведение имеет место при более высоких значениях массы бозона Хиггса, начиная с низшего (по данным СМВ) ограничения для $M_{\mathrm{H}} \simeq 135.6$ ГэВ примерно до 160 ГэВ. В этой области мы имеем семейство метастабильных вакуумов с $\widehat{V}>0$. Примером служит график для нижнего предела $M_{\mathrm{H}}=135.62$ ГэВ, изображенный на рис. 9 . Несмотря на мелкий характер потенциальной ямы этого вакуума, небольшой максимум потенциала порождает в соответствии с (32) острый вероятностный пик для начального значения поля инфлатона. Это связано с чрезвычайно малым значением $\widehat{V} / M_{\mathrm{P}}^{4} \sim 10^{-11}$, обратная степень которого порождает быстро изменяющуюся экспоненту в (32). Положение этого пика предшествует инфляционной стадии для базового возмущения СМВ, отвечающего $N=60$ (также обозначенного штриховыми линиями на рис. 9).

Для еще бо́льших $M_{\mathrm{H}}$ метастабильные вакуумы заменяются на отрицательный склон потенциала, который безостановочно падает до нуля при больших $t$, по меньшей мере в пределах применимости теории возмущений модели (см. рис. 7), поэтому для больших $M_{\mathrm{H}}$, близких к верхнему пределу 185 ГэВ, вероятностный пик в (32) оказывается отделенным от непертурбативной области сверхпланковских масштабов благодаря быстрому стремлению $\widehat{V} \sim \lambda / \xi^{2}$ к нулю. Это в свою очередь следует из того, что $\xi(t)$ растет много быстрее чем $\lambda(t)$, когда обе константы начинают приближаться к их полюсу Ландау [29].

\footnotetext{
5) Другая интересная область значений $M_{\mathrm{H}}$ лежит ниже порога нестабильности $M_{\mathrm{H}}^{\text {inst }}$, где $\widehat{V}$ становится отрицательным в “истинном" высокоэнергетическом вакууме. Как отмечалось в предыдущем разделе, туннелирующее состояние запрещает апериодические решения эффективных уравнений с $H^{2}<0$, которые не могут вносить вклад в квантовый ансамбль моделей с лоренцевой сигнатурой, поэтому эта область квазиклассически запрещена не только в соответствии с условиями нестабильности, но также противоречит туннелирующему предписанию.
} 


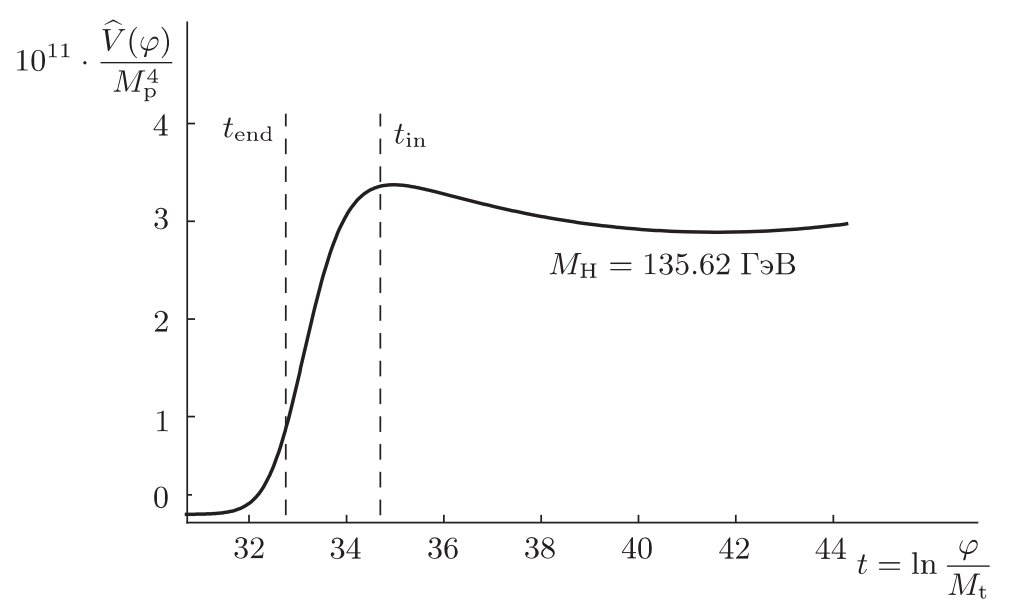

Рис. 9. Потенциал инфлатона при наименьшем значении $M_{\mathrm{H}}$, совместном с данными СМВ, обладающий метастабильным вакуумом при $t \simeq 42$ [29].

Положение $\varphi_{0}$ вероятностного пика и его квантовая ширина могут быть найдены в аналитической форме, и их вычисление показывает критическую роль бегущей константы $\mathbf{A}_{\mathrm{I}}(t)$ в формировании начальных условий инфляции. Действительно, показатель туннелирующего распределения (32) в случае $M_{\mathrm{P}}^{2} / \xi \varphi^{2} \ll 1$ имеет вид $\Gamma_{-}(\varphi)=24 \pi^{2} M_{\mathrm{P}}^{4} / \widehat{V}(\varphi)$ и в силу РГ-уравнений (35) обладает экстремумом, удовлетворяющим уравнению

$$
\varphi \frac{d \Gamma}{d \varphi}=\frac{d \Gamma}{d t}=-\frac{6 \xi^{2}}{\lambda}\left(\mathbf{A}_{\mathrm{I}}+\frac{64 \pi^{2} M_{\mathrm{P}}^{2}}{\xi Z^{2} \varphi^{2}}\right)=0,
$$

где мы снова пренебрегаем членами высокого порядка по $M_{\mathrm{P}}^{2} / \xi Z^{2} \varphi^{2}$ и $\mathbf{A}_{\mathrm{I}} / 64 \pi^{2}$ (выходящими за пределы однопетлевого приближения). Здесь $\mathbf{A}_{\text {I }}$ представляет собой аномальный скейлинг, введенный в соотношениях (36) и (37); эта величина должна быть отрицательна для существования решения

$$
\varphi_{0}^{2}=-\left.\frac{64 \pi^{2} M_{\mathrm{P}}^{2}}{\xi \mathbf{A}_{\mathrm{I}} Z^{2}}\right|_{t=t_{0}}
$$

уравнения, определяющего вероятностный пик. Как показано в работах [29], [30], эта величина в самом деле отрицательна. В совместной с данными СМВ области значений $M_{\mathrm{H}}$ она принимает начальные значения в интервале $-36 \lesssim \mathbf{A}_{\mathrm{I}}(0) \lesssim-23$ на EW-масштабе и достигает малых, но все еще отрицательных значений в интервале $-11 \lesssim \mathbf{A}_{\mathrm{I}}\left(t_{\text {end }}\right) \lesssim-2$ на масштабе инфляции. Также РГ-бег $\mathbf{A}_{\mathrm{I}}(t)$ и $Z(t)$ является очень медленным, имеет двухпетлевой порядок, и длительность инфляции очень короткая: $t_{0} \sim t_{\text {in }} \simeq t_{\text {end }}+2$ [29], [30], поэтому $\mathbf{A}_{\mathrm{I}}\left(t_{0}\right) \simeq \mathbf{A}_{\mathrm{I}}\left(t_{\text {end }}\right)$. Эти оценки применимы также к $\mathbf{A}_{\mathrm{I}}\left(t_{0}\right)$. В результате вторая производная туннелирующего действия положительна и очень велика, $d^{2} \Gamma_{-} / d t^{2} \simeq-\left(12 \xi^{2} / \lambda\right) \mathbf{A}_{\mathrm{I}} \gg 1$, что приводит к чрезвычайно малой квантовой ширине вероятностного пика:

$$
\frac{\Delta \varphi^{2}}{\varphi_{0}^{2}}=-\left.\frac{\lambda}{12 \xi^{2}} \frac{1}{\mathbf{A}_{\mathrm{I}}}\right|_{t=t_{0}} \sim 10^{-10} .
$$


Эта ширина в $\left(24 \pi^{2} /\left|\mathbf{A}_{\mathrm{I}}\right|\right)^{1 / 2}$ раз (один порядок величины) больше возмущений СМВ на базовой длине волны $k^{-1}=500$ Мпк (которую мы выбираем соответствующей $N=60$ ). Точку $\varphi_{\text {in }}$ пересечения горизонта этим возмущением (и волнами СМВ с другими $N$ ) легко получить из уравнения (38), которое в силу соотношения $\mathbf{A}_{\mathrm{I}}\left(t_{0}\right) \simeq$ $\mathbf{A}_{\mathrm{I}}\left(t_{\text {end }}\right)$ принимает вид

$$
\frac{\varphi_{\mathrm{in}}^{2}}{\varphi_{0}^{2}}=1-\exp \left(-N \frac{\left|\mathbf{A}_{\mathrm{I}}\left(t_{\text {end }}\right)\right|}{48 \pi^{2}}\right) .
$$

Это равенство указывает, что для длин волн, больших чем базовая, момент пересечения горизонта приближается к моменту "рождения" вселенной, но всегда остается хронологически более поздним, как это и должно быть.

\section{6. ЗАКЛЮЧЕНИЕ}

Мы построили туннелирующее квантовое состояние вселенной, основанное на интеграле по путям, для микроканонического ансамбля в космологии. В случае тяжелых массивных квантовых полей это состояние существует в неограниченной положительной области космологической постоянной, в отличие от тепловых "no-boundary" состояний Хартла-Хокинга для безмассовых конформных полей (космология СFT), обсуждавшихся в статьях [15], [16]. Ансамбль туннелирующих состояний ограничен обратным значением коэффициента топологического члена в полной конформной аномалии. Также, в отличие от случая "no-boundary", туннелирующее состояние оказывается свободным от радиации.

Статус туннелирующего состояния по сравнению с состояниями "no-boundary" является весьма замысловатым. Фактически формальный интеграл по путям (12) представляет собой преобразованный вариант микроканонического интеграла по лоренцевым метрикам, в котором интегрирование по функции хода осуществляется вдоль мнимой оси от $-i \infty$ до $+i \infty$ [16]. Отсутствие периодических решений для точек стационарности функционального интеграла (12) с лоренцевой сигнатурой приводит к необходимости деформировать контур интегрирования по $N$ в контур на комплексной плоскости так, что он пересекает вещественную ось в точках $N=+1$ или $N=-1$, которые порождают "no-boundary" состояние или туннелирующее состояние соответственно. Можно показать, что термическая "no-boundary" часть статистической суммы [15] не аналитична в полной комплексной плоскости значений $N$. Области $N>0$ и $N<0$ разделены бесконечной последовательностью полюсов этой части статистической суммы, плотно заполняющих мнимую ось $N$, поэтому контур интегрирования, проходящий через обе точки $N= \pm 1$, не существует, и "no-boundary" и туннелирующее состояния не могут быть получены аналитическим продолжением одно из другого ${ }^{6)}$. Они представляют разные решения (квантовые состояния) уравнения Уилера-де Витта.

\footnotetext{
6) Ситуация остается такой же и в случае вакуумного состояния "no-boundary", когда исчезающая термическая часть эффективного действия не может служить препятствием для аналитического продолжения в комплексной плоскости переменной $N$. Действительно, любой контур интегрирования от $-i \infty \mathrm{k}+i \infty$ пересекает вещественную ось нечетное число раз, в результате выживает вклад только одного такого пересечения, поскольку любые две (калибровочно-эквивалентные) седловые точки, проходимые в противоположных направлениях, дают взаимоуничтожающиеся вклады.
} 
В соответствии с обсуждением в п. 3.1 туннелирующее состояние для космологии CFT может быть альтернативным образом определено без аналитического продолжения из области положительной функции хода, но оказывается подавленным бесконечным положительным действием соответствующего вакуумного инстантона $S^{4}$. Такое динамическое подавление вообще бросает тень сомнения на существование туннелирующего состояния в квантовой космологии, потому что в реалистической космологии включение одной единственной безмассовой конформной частицы (типа фотона) может его разрушить. Однако мы предпочитаем не быть столь категоричными, поскольку регуляризация с помощью обрезания, упомянутая в п. 3.1, все еще позволяет проложить мостик для аналитического продолжения между областями положительной и отрицательной функции хода, причем результат такого продолжения остается открытой проблемой.

Для тяжелых квантовых полей формулировка интеграла по путям для туннелирующего состояния допускает непротиворечивую схему перенормировок и РГ-пересуммирование, которое согласно серии недавних работ [27]-[31] оказывается очень эффективным в космологии. По этой причине мы применили полученное туннелирующее распределение к недавно рассмотренной модели инфляции, генрируемой бозоном Хиггса СM, неминимально взаимодействующим с кривизной пространства-времени, в случае модели, в динамике которой преобладают тяжелые массивные частицы. Таким образом был получен полный космологический сценарий, охватывающий образование начальных условий динамики инфляционного фона (в форме острого вероятностного пика в распределении поля инфлатона) и последующую генерацию возмущений СМВ на этом фоне. Как было показано в работах [29], [30], сравнение амплитуды СMB и спектрального индекса с данными зонда WMAP налагает ограничения на допустимую область значений массы бозона Хиггса. Замечательно, что они оказываются совместными с широко признанными ограничениями стабильности EW-вакуума и соображениями применимости теории возмущений. Интересно, что поведение бегущего аномального скейлинга $\mathbf{A}_{\mathrm{I}}(t)<0$, которое критически важно для этих ограничений, также гарантирует существование полученного нами вероятностного пика [20]. Квантовая ширина этого пика на один порядок величины превосходит амплитуду спектра СMB на базовой длине волны, что может повлечь интересные следствия, которые можно наблюдать в эксперименте. К сожалению, эта квантовая ширина вряд ли является измеримой, потому что она соответствует возмущению с бесконечной длиной волны (формальный предел $N \rightarrow \infty$ в (39)), но опосредованный эффект такого квантового дрожания космологического фона заслуживает дальнейшего изучения.

Подводя итог, отметим, что полученные результаты предлагают убедительное объединение квантовой космологии с феноменологией частиц СМ, теорией инфляции и наблюдениями СМВ. Они поддерживают гипотезу, что должным образом расширенная CM [37], [38] может быть непротиворечивой квантовой теорией вплоть до квантовой гравитации и, возможно, позволяет объяснить фундаментальные основы всех главных явлений в ранней и современной космологии.

Благодарности. Автор выражает искреннюю признательность А. Ю. Каменщику, К. Киферу, А. А. Старобинскому и К. Штейнваксу за плодотворное сотрудничество, обзор результатов которого предлагается в настоящей работе. Автор бла- 
годарит за поддержку со стороны DFG (грант 436 RUS 17/3/07) во время его визита в университет г. Кельна, Германия, а также со стороны РФФИ (грант № 11-02-00512) и выражает благодарность Лаборатории теоретической физики университета г. Тур, Франция, за гостеприимство.

\section{Список литературы}

[1] J. B. Hartle, S. W. Hawking, Phys. Rev. D, 28:12 (1983), 2960-2975; S. W. Hawking, Nucl. Phys. B, 239:1 (1984), 257-276.

[2] А. Д. Линде, ЖЭЭФ, 87:2 (1984), 369-374; А. D. Linde, Lett. Nuovo Cimento, 39 (1984), 401-405; В.А. Рубаков, Писъма в ЖКЭТФ, 39:2 (1984), 89-91; Ю.Б. Зельдович, А. А. Старобинский, Писъма в астроном. ж., 10:5 (1984), 323-328.

[3] A. Vilenkin, Phys. Rev. D, 30:2 (1984), 509-511.

[4] C. Kiefer, Quantum Gravity, International Series of Monographs on Physics, 136, Oxford Univ. Press, Oxford, 2007.

[5] A. Vilenkin, Phys. Rev. D, 50:4 (1994), 2581-2594, arXiv: gr-qc/9403010.

[6] S. Coleman, Nucl. Phys. B, 310:3-4 (1988), 643-668.

[7] A. Vilenkin, Phys. Rev. D, 37:4 (1988), 888-897; T. Vachaspati, A. Vilenkin, Phys. Rev. D, 37:4 (1988), 898-903.

[8] C. Kiefer, Phys. Rev. D, 46:4 (1992), 1658-1670.

[9] A. Vilenkin, Phys.Rev. D, 58:6 (1998), 067301, 3 pp., arXiv: gr-qc/9804051; AIP Conf. Proc., 478 (1999), 23-29, arXiv: gr-qc/9812027.

[10] A. O. Barvinsky, A. Y. Kamenshchik, Class. Quantum Grav., 7:12 (1990), 2285-2294.

[11] A. O. Barvinsky, Phys. Rep., 230:5-6 (1993), 237-367.

[12] A. O. Barvinsky, Phys. Rev. D, 50:8 (1994), 5115-5124, arXiv: gr-qc/9311023; A. O. Barvinsky, A. Yu. Kamenshchik, C. Kiefer, Nucl. Phys. B, 552:1-2 (1999), 420-444, arXiv: gr-qc/9901055.

[13] A. O. Barvinsky, A. Yu. Kamenshchik, Phys. Lett. B, 332:3-4 (1994), 270-276, arXiv: gr-qc/9404062.

[14] A. O. Barvinsky, A. Yu. Kamenshchik, Nucl. Phys. B, 532:1-2 (1998), 339-360, arXiv: hep-th/9803052.

[15] A. O. Barvinsky, A. Yu. Kamenshchik, JCAP, 09 (2006), 014, arXiv: hep-th/0605132; Phys. Rev. D, 74:12 (2006), 121502, 5 pp., arXiv: hep-th/0611206.

[16] A. O. Barvinsky, Phys. Rev. Lett., 99:7 (2007), 071301, 4 pp., arXiv: 0704.0083.

[17] A. O. Barvinsky, C. Deffayet, A. Yu. Kamenshchik, JCAP, 05 (2008), 020, 18 pp., arXiv: 0801.2063.

[18] M. V. Fischetti, J. B. Hartle, B. L. Hu, Phys. Rev. D, 20:8 (1979), 1757-1771.

[19] A. A. Starobinsky, Phys. Lett. B, 91:1 (1980), 99-102.

[20] A. O. Barvinsky, A. Yu. Kamenshchik, C. Kiefer, C. F. Steinwachs, Phys. Rev. D, 81:4 (2010), 043530, 9 pp., arXiv: 0911.1408.

[21] B. L. Spokoiny, Phys. Lett. B, 147:1-3 (1984), 39-43; R. Fakir, W. G. Unruh, Phys. Rev. D, 41:6 (1990), 1783-1791; D. S. Salopek, J.R. Bond, J. M. Bardeen, Phys. Rev. D, 40:6 (1989), 1753-1788.

[22] E. Komatsu, T. Futamase, Phys. Rev. D, 59:6 (1999), 0064029, 7 pp., arXiv: astro-ph/9901127.

[23] F. L. Bezrukov, M. Shaposhnikov, Phys. Lett. B, 659:3 (2008), 703-706, arXiv: 0710.3755.

[24] A. O. Barvinsky, A. Yu. Kamenshchik, A. A. Starobinsky, JCAP, 11 (2008), 021, 13 pp., arXiv: 0809.2104.

[25] J. Garcia-Bellido, D. G. Figueroa, J. Rubio, Phys. Rev. D, 79:6 (2009), 063531, 22 pp., arXiv: 0812.4624. 
[26] F. L. Bezrukov, A. Magnin, M. Shaposhnikov, Phys. Lett. B, 675:1 (2009), 88-92, arXiv: 0812.4950 .

[27] A. De Simone, M. P. Hertzberg, F. Wilczek, Phys. Lett. B, 678 (2009), 1-8, arXiv: 0812.4946 .

[28] F. Bezrukov, M. Shaposhnikov, JHEP, 07 (2009), 089, 24 pp., arXiv: 0904.1537.

[29] A. O. Barvinsky, A. Yu. Kamenshchik, A. A. Starobinsky, C. Steinwachs, JCAP, 12 (2009), 003, 28 pp., arXiv: 0904.1698.

[30] A. O. Barvinsky, A. Yu. Kamenshchik, C. Kiefer, A. A. Starobinsky, C. Steinwachs, Higgs boson, renormalization group, and naturalness in cosmology, arXiv: 0910.1041.

[31] T. E. Clark, B. Liu, S. T. Love, T. ter Veldhuis, Phys. Rev. D, 80:7 (2009), 075019, 13 pp., arXiv: 0906.5595.

[32] A. O. Barvinsky, C. Deffayet, A. Yu. Kamenshchik, JCAP, 05 (2010), 034, 20 pp., arXiv: 0912.4604 .

[33] A. O. Barvinsky, D. V. Nesterov, Nucl. Phys. B, 608:1-2 (2001), 333-374, arXiv: gr-qc/0008062.

[34] G. Hinshaw et al., Astrophys. J. Suppl., 180:2 (2009), 225-245, arXiv: 0803.0732; E. Komatsu et al., Astrophys. J. Suppl., 180:2 (2009), 330-376, arXiv: 0803.0547.

[35] J. R. Espinosa, G. F. Giudice, A. Riotto, JCAP, 05 (2008), 002, 24 pp., arXiv: 0710.2484.

[36] M. Sher, Phys. Rep., 179:5-6 (1989), 273-418.

[37] T. Asaka, S. Blanchet, M. Shaposhnikov, Phys. Lett. B, 631:4 (2005), 151-156, arXiv: hep-ph/0503065; T. Asaka, M. Shaposhnikov, Phys. Lett. B, 620:1-2 (2005), 17-26, arXiv: hep-ph/0505013.

[38] M. Shaposhnikov, D. Zenháusern, Phys. Lett. B, 671:1 (2009), 18-1927, arXiv: 0809.3395. 